\title{
Multipactor simulations in superconducting cavities and power couplers
}

\author{
G. Devanz* \\ CEA/DSM/DAPNIA, Bâtiment 701, l'Orme des merisiers, Gif-sur-Yvette F91191, France
}

(Received 6 October 2000; published 5 January 2001)

\begin{abstract}
A new computer code for simulation of multipacting phenomenon in axisymmetrical radio frequency structures is presented. The existence of resonant electron trajectories can lead to electron multiplication under given field and surface conditions and can damage these accelerator components or prevent their use. The goal of the simulation is to detect such occurrences. Therefore, large parameter sets have to be tested; a parallel version of the code has been developed to reduce computing time while dealing with highly demanding cases in terms of accuracy and parameter sweeping. Both superconducting cavities and power coupler components have been studied. Simulation results are compared with experimental measurements.
\end{abstract}

DOI: 10.1103/PhysRevSTAB.4.012001

PACS numbers: 52.80.Vp, 52.80.Pi, 52.20.Dq

\section{INTRODUCTION}

High power $\mathrm{rf}$ accelerator components under vacuum can be plagued by a parasitic phenomenon called multipactor (MP). Its occurrence is related to the existence of resonant trajectories in the structure, depending on the electromagnetic (EM) field distribution and the geometry of the structure. These trajectories present $p, p \geq 1 \mathrm{im}-$ pact points at which electrons hit the walls in synchronism with the rf field. Materials constituting the rf structure present a secondary electron emission (SEE) coefficient $\delta$, varying with $e^{-}$impact energy $W_{i}$, and possibly greater than 1 between $E_{1}$ and $E_{2}$ characteristic energies. For standard materials used in accelerator rf technology, $E_{1}$ ranges typically from several tens to a few hundreds $\mathrm{eV}$, while $E_{2}$ ranges from a few hundreds to a few thousands $\mathrm{eV}$. If $\delta$ is greater than 1 for the electron impact energy of the resonant trajectories, the number of electrons $N_{e}$ can grow exponentially. In reality, this growth is limited by interaction with the residual and desorbed gases and by space charge in the electron bunches created in the process. The consequence of multipactor can range from a lengthening of the $\mathrm{rf}$ conditioning process to the destruction of the component.

For a given problem description, MP is a three parameter problem, since it depends on the field level $E$, the electron starting position $s_{0}$, and phase $\phi_{0}$. Although the resonance can be predicted analytically for simple shapes such as parallel plates, MP occurrence has to be investigated by simulation means in the case of a realistic design. In this paper, we present a numerical code capable of computing electron trajectories in order to detect and study multipactor phenomenon in 2D axisymmetric rf structures. The field of application comprises accelerating cavities, especially superconducting cavities, power couplers, transmission lines, and rf power windows. Studies of real cases will be pre-

*Email address: gdevanz@cea.fr sented for each of these items, and a particular stress will be put on several rf window designs for which we achieved a better understanding of the MP phenomenon.

\section{CODE DESIGN}

\section{A. Modeling}

The MUPAC code has been developed to simulate MP in axisymmetrical rf structures, working in any wave propagation regime. Problem geometry and EM fields are imported from SUPERFISH [1]. SEE properties of materials can be read from user data files or specified with the parameters $\delta_{\max }$, the maximum of $\delta$, and $E_{\max }$, the $e^{-}$impact energy at which this maximum is obtained.

Multipactor occurrence is usually stated when resonant trajectories are detected. For traveling wave devices, this definition can still be useful if the geometry is $L_{z}$ periodic along the propagation direction $z$, and resonance is to be searched considering the axial position modulo $L_{z}$ as done in [2]. However, in the general case, the geometry displays no axial periodicity. rf windows illustrate this configuration, although showing harmful $e^{-}$activity. Therefore, the evolution of $N_{e}$ is monitored for each parameter set $\left(E, s_{0}, \phi_{0}\right)$; the $s_{0}$ parameter should be swept along the whole rf contour to detect every possible source of multipactor. Calculations are done for every $\phi_{0}$ value, even if it corresponds to a decelerating field, since secondary $e^{-}$ have an initial kinetic energy and may nevertheless escape the surface.

The code performs the integration of the equation of motion of a single electron; for each impact $i$ occurring at the curvilinear abscissa $s_{i}$ and phase $\phi_{i}$, the electron kinetic energy $E_{i}$ and angle of incidence on the surface $\theta_{i}$ determine the electron multiplication factor $N_{i}=\delta\left(s_{i}, E_{i}, \theta_{i}\right)$. The current electron number at the $j$ th impact is then computed according to $N_{e}(j)=N_{1} \times \cdots \times N_{j}$ and a new electron is emitted. Computation is stopped whether $N_{e}$ goes out of user defined bounds $N_{e}^{\min }, N_{e}^{\max }$ or the limit $i_{\max }$ on the number of impact was reached. A normalized 
current $\tilde{I}=I / \omega_{\mathrm{rf}} e=N_{e}(i) /\left(\phi_{i+1}-\phi_{i}\right)$ is computed between two impacts, of which the code keeps track. No space charge nor any $e^{-}$- gas or plasma-interaction is taken into account.

\section{B. Design overview}

The main role of the code is to integrate the relativistic equation of motion of electrons in time-varying EM fields. Since the electric field's local amplitude can range from almost zero to several $\mathrm{MV} / \mathrm{m}$ depending on the problems, the integration algorithm has to be extremely versatile and reliable. We have chosen a 4th-5th order adaptive Runge-Kutta method described in [3]. Its accuracy is kept tunable by the user. Since precise determination of impact position and phase is required in MP calculations, and due to the grid representation of EM fields, the latter have to be extrapolated near the geometric boundaries and interpolated everywhere else. Several tests have been performed to choose the approximation technique, which lead to the conclusion that it was more efficient to choose a finer grid and bilinear interpolation rather than a looser grid and higher order interpolation, both in terms of accuracy and speed. Extensive use is made of lookup tables both for approximation of the fields and localization of the electrons in the geometry that could yield a global speedup factor of 2 for a standard simulation.

The problem geometry is internally stored as in a CAD software; therefore, impacts can be analytically computed to achieve the high accuracy needed.

\section{Parallel version}

A typical application of the code to simulate an rf window leads to $10^{6}-10^{8}$ full trajectory computations corresponding to several tens of hours on a $500 \mathrm{MHz}$ personal computer. A parallel version of the code was developed to run on a network PC or workstations using the parallel virtual machine (PVM) library [4] to reduce computing time without needing supercomputer access. We used a masterslave scheme, in which a master program distributes parameter sets to every slave. As soon as a slave finishes the computation, it returns its result and immediately gets a new parameter set. This permits one to achieve load balancing since a faster or unloaded CPU will compute many more parameter sets than a slow or loaded one.

\section{SUPERCONDUCTING CAVITIES}

The code can be used to simulate MP in superconducting rf (Srf) cavities. Several successful comparisons between MUPAC simulations and $700 \mathrm{MHz}$ high intensity proton linac cavity tests results have been reported in [5]. We give here an example of two different single cell cavities tested at $1.8 \mathrm{~K}$ at Saclay, which have gone through the same fabrication and cleaning procedures. The first is an elliptical cav-

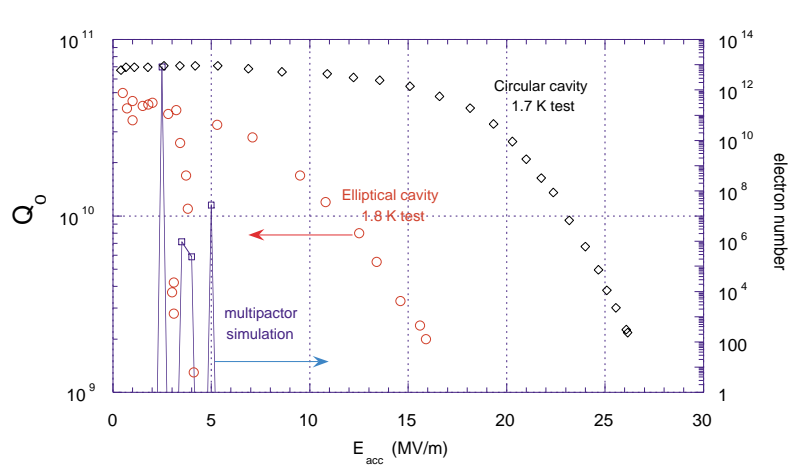

FIG. 1. (Color) Comparison between $700 \mathrm{MHz}$ circular and elliptical cavity cold test results and MP simulation.

ity for which an electronic activity was detected at several accelerating field values as shown in Fig. 1. Drops of the quality factor indicate rf power consumption during electron activity. MUPAC simulation results are in good agreement with the measurements. The second cavity presents a circular shape at the equator, and could be tested to higher accelerating gradients without electron activity. Simulations could not detect any multipacting behavior either.

\section{POWER COUPLERS}

Among various high power rf components used to drive rf accelerators, coaxial power couplers are known to be prone to multipact, and several schemes have been proposed to control and possibly suppress it, complicating its technical design and eventually lowering its reliability. The rf window, which should both play the role of a vacuum barrier and transmit rf power, appears to be mostly sensitive to multipactor phenomena. This is due to a large extent to the fact that alumina is generally used, exhibiting a high SEE coefficient. We will focus on several types of coaxial rf windows for which a MP study was carried out numerically and compared to experimentally observed electron activity. These windows have been designed in the framework of the Tesla Test Facility (TTF) power coupler research and development program and work around $1.3 \mathrm{GHz}$ frequency.

\section{A. The half wavelength window}

A $\lambda / 2$ disk coaxial window described in [6] was tested on our $1.3 \mathrm{GHz}$ power bench where electron activity can be measured by means of electron pickups installed near the ceramic disk. MP simulations and experimental electron activity when varying rf power are compared in Fig. 2. The highest simulated MP barriers match the experimental results, as far as the power levels are concerned. The latter correspond to multipacting levels found for a bare coaxial line with the same dimensions. The interpretation is that multipacting $e-$ drift along the coaxial line and eventually impinge on the ceramic disk, enhancing the coax MP behavior. 


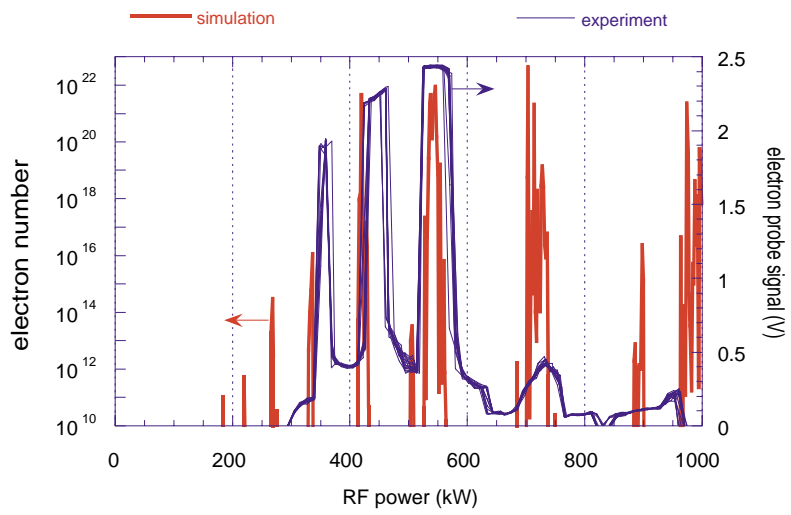

FIG. 2. (Color) Measured and simulated electron activity on the upstream side of the $\lambda / 2$ window.

\section{B. Traveling wave window - cavity type}

The multipactor behavior of the traveling wave (TW) window (details concerning design parameters and experimental tests can be found in [7]) has been studied with MUPAC. A quarter of the geometry is represented in Fig. 3, where a curvilinear abscissa is indicated for further reference.

The window simulation is performed in four typical surface processing conditions for the conducting surfaces and the ceramic disk, respectively ( $\delta_{\max }$ are provided, enclosed in parentheses). During the conditioning process, electron dose deposition on materials lowers their SEE function, as shown experimentally on $\mathrm{Cu}$ in [8].

Case A: nonclean copper (1.8) and bare $\mathrm{Al}_{2} \mathrm{O}_{3}$ (2.7). This parameter setting can describe a window at the beginning of the rf power tests. Although our ceramic disk was TiN coated, this fictive case is useful to evaluate the efficiency of the design in terms of MP and the influence of the coating.

Case B: conditioned copper (1.3) and bare $\mathrm{Al}_{2} \mathrm{O}_{3}$ (2.7).

Case C: nonclean copper (1.8) and TiN coated $\mathrm{Al}_{2} \mathrm{O}_{3}$ (1.1).

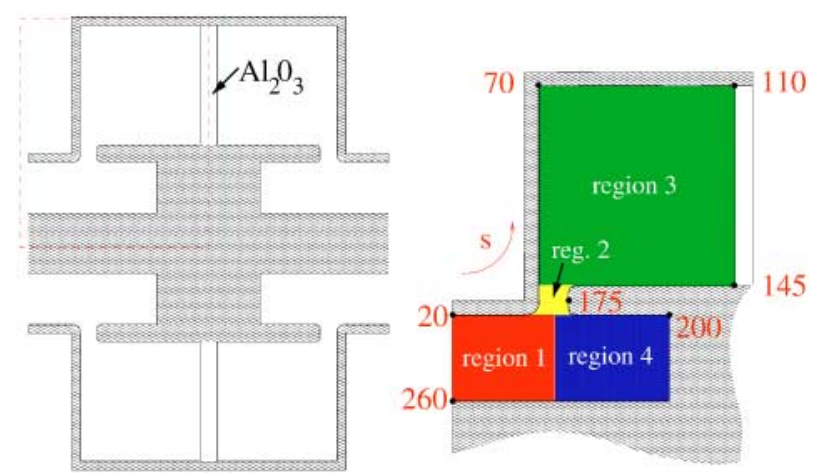

FIG. 3. (Color) Profile of the cavity-type TW window, with curvilinear abscissa $(s)$ reference.
Case D: conditioned copper (1.3) and TiN coated $\mathrm{Al}_{2} \mathrm{O}_{3}$ (1.1). This should well describe the window at the end of the conditioning process.

Sweeping electron starting locations on the upstream side contour reveal different MP behaviors as shown in Fig. 4. Region 1 is the coaxial part and exhibits usual coax MP barriers. No specific MP can exist in the gap region (region 2) since electric fields are the strongest of the structure, and, for a given rf input power, electron impact energies are relatively higher than elsewhere in the window, above $E_{2}$ to a large extent. This region can rather act as a source of electrons, and simulation shows that $e^{-}$ emitted from the gap are either deflected in the coax line or accelerated towards the cavity. Depending on the rf power, these electrons participate in the MP behavior of either of these regions. The cavity region (region 3 ) shows a homogeneous behavior with respect to the starting position of the electrons, and multipacting is rejected to the higher powers, due to the reduced values of the electric field in the ceramic area. One exception is the upper left corner where the $E$ field is too weak to even accelerate electrons to multipacting areas. Electron activity with respect to $\mathrm{rf}$ power occurs mainly at $900 \mathrm{~kW}$, but emerges above a continuous background. The $900 \mathrm{~kW}$ MP corresponds to a resonant phenomenon, whereas the surrounding activity is associated with long living trajectories with an average impact energy in the range $E_{1}-E_{2}$ but no resonant behavior. The multiplication phenomenon is slightly enhanced in the

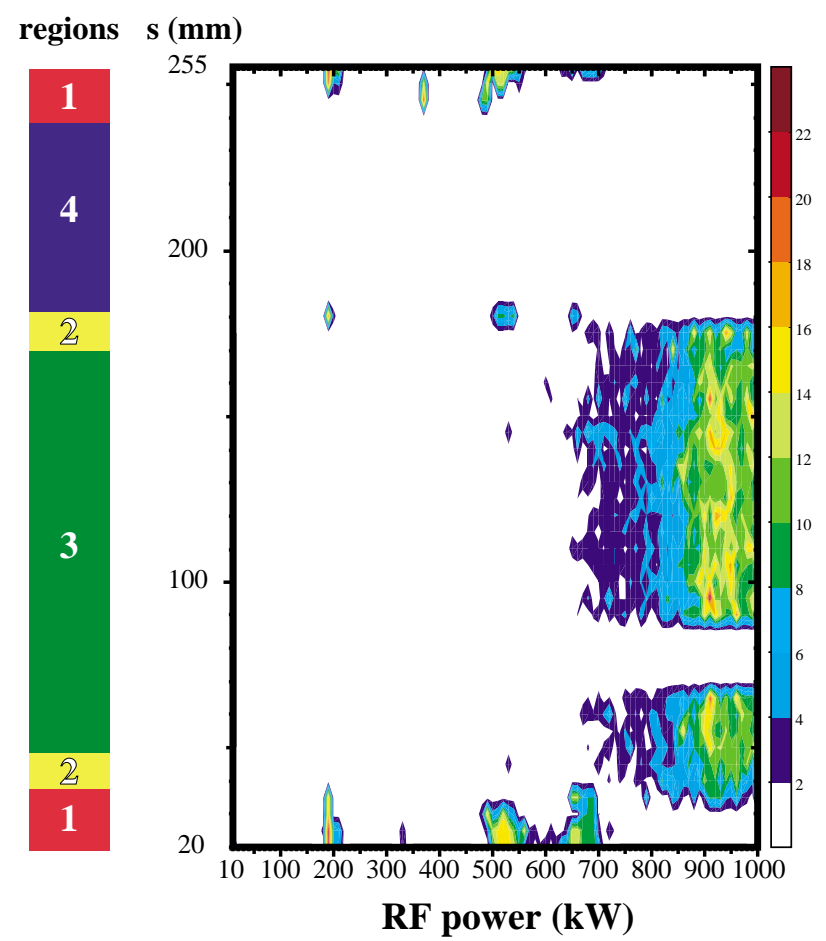

FIG. 4. (Color) Simulated $\log \left(N_{e}\right)$ as a function of rf input power and electron starting position $s$ for the cavity-type TW window. 


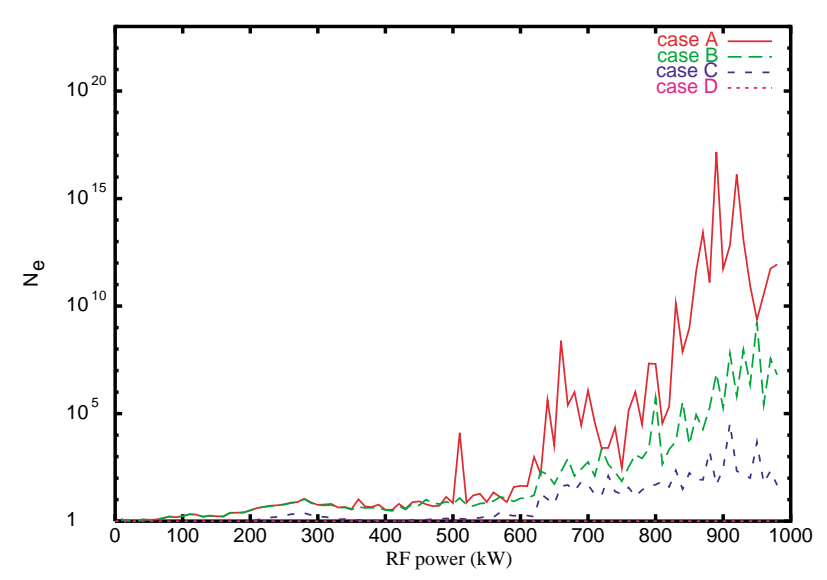

FIG. 5. (Color) Simulated $N_{e}$ as a function of rf input power for material configurations defined earlier, for trajectories starting inside the cavity.

vicinity of the junction between the inner conductor and the ceramic disk.

The recessed part of the coaxial line (region 4) does not show any multipacting in the power range of interest. The electric field is so low that impact energies higher than $E_{1}$ cannot be reached.

The effect of conditioning and TiN deposition can be seen in Fig. 5. Trajectories are all started at $s_{0}=145$, inside the cavity. Conditioning of copper alone is not sufficient to suppress electron activity. TiN coating successfully reduces MP and, in Case $\mathrm{D}, e^{-}$multiplication is inhibited thanks to the combined effect of both SEE $\delta$ reductions.

Because of the spread in minimum reflection frequency of the rf components of the test bench, it was difficult to know the voltage standing wave radio (VSWR) and the standing wave pattern position in the window with precision during the experiments. The increase of electron activity with rf power above $700 \mathrm{~kW}$ described earlier was observed experimentally, but on the downstream side of the window. Since the simulation was done for a pure TW case, the difference in VSWR might explain this discrepancy.

\section{Traveling wave window-iris type}

Another design is proposed to overcome the difficulty of cleaning the preceding window according to the cleanness standard required for the operation of TTF high gradient Srf cavities. The rf concept is based on [9], and leads to a simple design shown in Fig. 6.

The same simulation procedure as that of Sec. IV B was applied and leads to results shown in Fig. 7 for Case A.

Electron activity is generated from the iris region and a $70 \mathrm{~kW}$ wide 2 points barrier is predicted around $600 \mathrm{~kW}$ input power. However, among trajectories started between the iris top and the outer conductor (region 2), those which stay on the iris are all fading away. Only those which

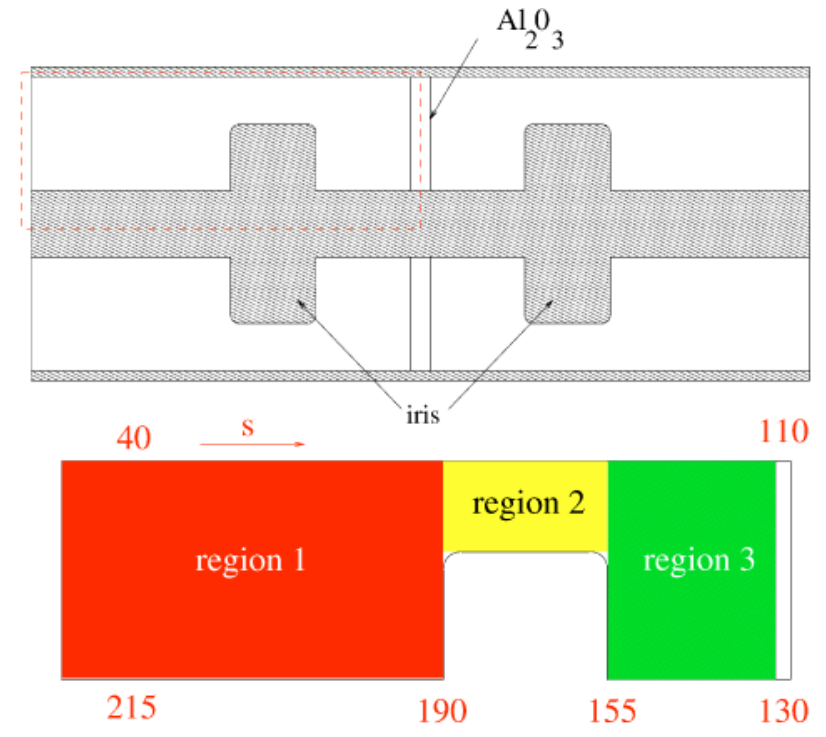

FIG. 6. (Color) Profile of the iris-type TW window, with curvilinear abscissa $(s)$ reference.

drift to the upstream side of the coax can become resonant around $600 \mathrm{~kW}$, and those shifting to the downstream side can multipact around $350 \mathrm{~kW}$, in the region between the iris and the ceramic disk (region 3), but never hit the latter.

Simulations in Cases C and A yield the same results, implying that an SEE variation of the ceramic disk has no influence on electron activity. We can conclude from this observation that no harmful trajectory hits the dielectric

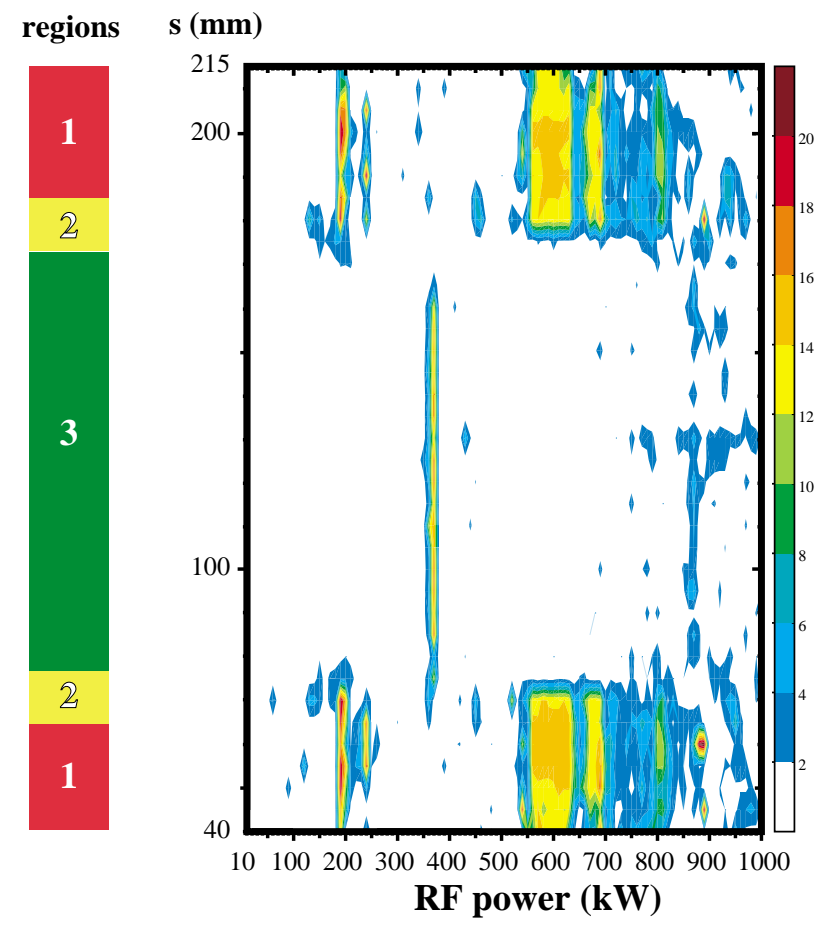

FIG. 7. (Color) Simulated $\log \left(N_{e}\right)$ as a function of rf input power and electron starting position $s$ for the iris-type TW window. 
disk. The first advantage is a reduced risk of charge buildup on the ceramic, and the second advantage is the possibility of circumventing the use of a TiN coating, thereby reducing $r f$ losses and fabrication costs of the window.

The particularity of this design resides in the fact that the iris locally creates a longitudinal variation of the fields which is responsible for a longitudinal focusing of the $e^{-}$ trajectories. If the accumulation point of the trajectory is kept at a distance from the ceramic, dangerous electron multiplication is avoided. The iris thus creates an electromagnetic trap for the electrons.

\section{CONCLUSION}

The MUPAC code was used to help in the analysis of experimental electron activity data where it proves both robustness and accuracy. The code was employed for superconducting cavity and coupler components simulations. In power $\mathrm{rf}$ windows, nonresonant, long living trajectories could be identified both experimentally and numerically, which could affect window conditioning as much as regular resonant multipactors do. MUPAC was also used to test and improve an rf window design in order to keep multipactor off the ceramic disk, thus minimizing the probability of window breakage.

\section{ACKNOWLEDGMENTS}

The author thanks J-L. Biarrote and H. Safa for providing him with experimental results on Srf cavities, and
M. Desmons, S. Chel, and C. Travier for coupler test bench data and useful discussions.

[1] J. Billen, SUPERFISH V 5.0 User Guide, LANL Report No. LA-UR-96-1834, 2000.

[2] E. Somersalo, P. Ylä-Oijala, D. Proch, and J. Sarvas, Part. Accel. 61, 107 (1998).

[3] W. H. Press, S. A. Teukolsky, W. T. Vetterling, and B. P. Flannery, Numerical Recipes in C (Cambridge University, Cambridge, England, 1997), 2nd ed.

[4] A. Geist, A. Beguelin, J. Dongarra, W. Jiang, R. Manchek, and V. Sunderam, ONRL Technical Report No. TM-12187, 1994.

[5] G. Devanz, in Proceedings of the European Particle Accelerator Conference, Vienna, 2000, http:/accelconf.web. cern.ch/AccelConf/e00/index.html

[6] S. Chel, M. Desmons, C. Travier, T. Garvey, P. Lepercq, and R. Panvier, in Proceedings of the 1999 Particle Accelerator Conference, New York, 1999 (IEEE, Piscataway, NJ, 1999), pp. 916-918.

[7] C. Travier, S. Chel, M. Desmons, and G. Devanz, in Proceedings of the 9th Workshop on RF Superconductivity, Santa Fe, NM, 1999 (LANL Report No. LA-13782-C, 2000).

[8] I. Bojko, N. Hilleret, and C. Scheurerlein, J. Vac. Sci. Technol. A 18, 972 (2000).

[9] Y. Kazakov, Budker Institute of Nuclear Physics Report No. 92-2, 1992. 